\title{
and
}

\section{Infecciones fúngicas en pacientes con COVID19}

\author{
Recibido: 1/11/20 Aceptado: 10/12/20
}

Fernando A. Messina', Emmanuel Marin'1, Mario Valerga², Roxana Depardo', Viviana Chediak², María de las Mercedes Romero ${ }^{1}$, Andrés Benchetrit ${ }^{4}$, Nicolás Lista ${ }^{5}$, Alejandra Rodríguez ${ }^{6}$, Cecilia Domínguez ${ }^{7}$, Belén Bouzas ${ }^{8}$, Eleonora Cunto ${ }^{9}$, Daniela Vaustat ${ }^{10}$ y Gabriela María Santiso ${ }^{1}$.

\section{RESUMEN}

En diciembre de 2019 se identificó en Wuhan, China, un nuevo coronavirus denominado SARS-CoV-2, agente causal de la epidemia de neumonía atípica COVID-2019, que el 11 de marzo de 2020 fue declarada pandemia por la OMS.

Hasta el 30 de septiembre de 2020, en Argentina fueron confirmados 751.001 casos y más de 16.937 muertes. La frecuencia y el impacto de las coinfecciones que afectan a los pacientes infectados por SARS-Cov-2 se ha estudiado junto con el avance de la pandemia. Entre las debidas a hongos se encuentran las fungemias por Candida sp, la aspergilosis invasora, las micosis sistémicas endémicas y la neumocistosis.

Presentamos las distintas coinfecciones micosisCOVID-19 que fueron asistidas en nuestra institución entre abril y septiembre de 2020, y se realiza un análisis de las características de estas infecciones en pacientes con y sin sida. En este período se internaron 2837 pacientes, 2287 tuvieron diagnóstico confirmado de COVID-19. La coinfección de COVID-19 con micosis pulmonares o sistémicas fue menor al $1 \%$.

Dieciocho pacientes presentaron infecciones fúngicas pulmonares o sistémicas. Ocho padecieron candidemias, cinco criptococosis meningeas, dos histoplasmosis, dos aspergilosis invasoras agudas probables y una aspergilosis pulmonar crónica.

La estadía prolongada en terapia intensiva facilitó las fungemias por Candida sp, los casos de histoplasmosis y criptococosis parecen relacionarse con la enfermedad avanzada por VIH y no con COVID-19.

\begin{abstract}
1 Unidad Micología Hospital de Infecciosas Francisco J. Muñiz, Centro de Referencia de la Ciudad Autónoma de Buenos Aires, Argentina.

2 Sala 35 Pabellón Koch/internación CoVID-19. Hospital de Infecciosas F. J. Muñiz, Buenos Aires, Argentina.

${ }^{3}$ Sala 3, Unidad de Cuidados Intensivos. Hospital de Infecciosas F. J. Muñiz, Buenos Aires, Argentina. ${ }^{4}$ Sala 21 y Pabellón Koch/COVID-19. Hospital de Infecciosas F. J. Muñiz, Buenos Aires, Argentina. ${ }^{5}$ Sala 2, Unidad de Cuidados Intensivos. Hospital de Infecciosas F. J. Muñiz, Buenos Aires, Argentina. ${ }^{6}$ Sala 1, Unidad de Cuidados Intensivos. Hospital de Infecciosas F. J. Muñiz, Buenos Aires, Argentina. ${ }^{7}$ Sala 32, Unidad de Cuidados Intensivos (internación COVID-19). Hospital de Infecciosas F. J. Muñiz, Buenos Aires, Argentina.

${ }^{8}$ División Análisis Clínicos, Hospital de Infecciosas F. J. Muñiz, Buenos Aires, Argentina.

${ }^{9}$ Departamento Atención Intensiva del Paciente Infeccioso Crítico (DAIPIC). Hospital de Infecciosas F. J. Muñiz, Buenos Aires, Argentina.

${ }^{10}$ Unidad de Bacteriología. Hospital de Infecciosas F. J. Muñiz, Buenos Aires, Argentina.

Autor para correspondencia: Fernando Messina. Uspallata 2272, C1282 CABA.

Email: fmessina35@gmail.com

No se recibió ninguna fuente de apoyo ni financiación. Los autores declaran no tener ningún conflicto de interés.
\end{abstract}

Los enfermos con un componente inflamatorio basal alto con neumonía grave por coronavirus se relacionan más con micosis invasoras que los enfermos VIH positivos con niveles bajos de LTCD4+.

Palabras clave: micosis y COVID-19, candidemia y COVID-19, SARS-CoV-2 y micosis y aspergilosis invasora. 


\section{Introducción}

Los coronavirus son virus envueltos cuyo genoma es RNA de simple cadena. Pertenecen a una gran familia de virus (Coronaviridae) que infectan aves y varios mamíferos, incluyendo camélidos, murciélagos, ratas, ratones, perros, gatos y humanos (1). En estos últimos, algunos miembros de esta familia son conocidos desde hace décadas por provocar síntomas de resfrío común. Los coronavirus han sido reconocidos como causantes de graves infecciones respiratorias e intestinales. En 2002, en la provincia de Guangdong, China (2), se inició un brote de Síndrome Respiratorio Agudo Severo (SARS) cuyo agente etiológico se extendió a cinco continentes, infectando 8098 personas y causando 774 muertes. En 2012, en la península arábica emergió otro coronavirus (MERS-CoV) que fue exportado a 27 países, causando 2494 infecciones y 88 muertes (3).

En diciembre de 2019 se identificó por primera vez en Wuhan, China un nuevo coronavirus previamente desconocido, denominado SARS-CoV-2, que es el agente causal de la epidemia de neumonía atípica (COVID-2019; CoronaVirus Disease 2019) (4). Debido a su velocidad de expansión y gravedad, el 11 de marzo de 2020 la Organización Mundial de la Salud (OMS) declaró la pandemia.

Desde diciembre de 2019, la pandemia de COVID-19 se ha propagado desde su epicentro en Wuhan, China, para infectar a más de 32 millones de personas, con más de 991.224 muertes asociadas con la enfermedad en todo el mundo (5).

En Argentina, el primer caso confirmado fue el 3 de marzo de 2020. Hasta el 30 de septiembre de 2020 en Argentina fueron confirmados 751.001 casos con más de 16.937 muertes (6).

Los síntomas más comunes son fiebre, tos, odinofagia y anosmia (7). Si bien el $81 \%$ de los pacientes presentan formas leves, un $14 \%$ serán graves y el $5 \%$ restante presentará cuadros críticos (insuficiencia respiratoria, choque séptico, y/o falla multiorgánica) (8). Los factores de riesgos relacionados a una mala evolución son la hipertensión, la diabetes, la obesidad y ser mayor de 75 años de edad (7).

Entre varias causas de morbilidad y mortalidad en pacientes con COVID-19, la frecuencia y el impacto de las coinfecciones se ha estudiado a medida que avanza la pandemia en los distintos países.
Las micosis no son consideradas en la actualidad infecciones infrecuentes que solo afectan a un número reducido de pacientes. En general estas se dividen entre las endémicas y las oportunistas. Las primeras pueden afectar tanto a huéspedes inmunocompentes como inmunocomprometidos. Las segundas afectan a un grupo heterogéneo de pacientes con diferentes tipos y grados de inmunocompromiso.

Entre las coinfecciones debidas a hongos que afectan a los pacientes infectados por SARS-CoV-2 podemos mencionar a las fungemias por Candida sp, la aspergilosis invasora, las micosis sistémicas endémicas y la neumocistosis.

La infección fúngica invasora puede ser subdiagnosticada si no se piensa en ella y no se normatiza la metodología diagnóstica. Todavía no se conoce exactamente cómo la coinfección micótica impacta en la evolución y por consecuencia en la mortalidad de estos pacientes en nuestro país. Solo se conocen datos de series europeas (9), asiáticas (10) o de Estados Unidos (11). El impacto de coinfección por influenza y aspergilosis invasora ha sido previamente descripto (12) y en algunos centros europeos alcanzó una mortalidad del $23 \%$. En el estudio de Schauwvlieghe (13), la tasa de mortalidad a tres meses de la influenza es del $51 \%$ cuando se asocia con Al y $28 \%$ sin Al. Además, la fisiopatología particular de COVID-19 también puede representar una comorbilidad con infección fúngica invasora (IFI). Las características agresivas del virus SARS-CoV-2 en el tejido pulmonar y las grandes lesiones alveolo-intersticiales bilaterales hacen que estos pacientes sean susceptibles de sufrir una IFI (14).

No existe evidencia en la actualidad de que personas con enfermedad $\mathrm{VIH}$ /sida tengan un mayor riesgo de contagio de COVID-19. Pero en el brote epidémico ocurrido en España se ha demostrado que los pacientes con sida evolucionaban a formas graves cuando el recuento de linfocitos TCD4+ era menor a 200 células/ $\mu \mathrm{L}$ o cuando la edad de los individuos superaba los 75 años, ya que en estas circunstancias se agregaban otras comorbilidades (15).

\section{Objetivos}

El objetivo de este trabajo es presentar las distintas coinfecciones micosis-COVID19 que fueron asistidas en nuestra institución desde el comienzo de esta pandemia hasta la fecha. También, describir la metodología 
diagnóstica, el tratamiento y la evolución de dichas micosis en el contexto de la infección por COVID-19.

\section{Materiales y métodos}

Se realizó un estudio retrospectivo, observacional y descriptivo donde se analizaron las historias clínicas de enfermos con micosis pulmonares o sistémicas que padecieron coronavirus, asistidos en el Hospital de Infecciosas F. J. Muñiz entre el 1 de abril y el 30 de septiembre de 2020. Además, se analizaron las diferencias entre los pacientes $\mathrm{VIH}$ positivos y negativos.

\section{Criterios de inclusión}

Todos los enfermos adultos (mayores de 18 años de edad) internados con micosis pulmonares o sistémicas diagnosticados en la unidad Micología del Hospital F. J. Muñiz con diagnóstico de coronavirus por PCR de hisopado nasofaríngeo.

\section{Criterios de exclusión}

Enfermos con diagnóstico de micosis pulmonares o sistémicas realizados en la unidad Micología del Hospital F. J. Muñiz con COVID-19 pero internados en otras instituciones.

\section{Diagnóstico}

Para el diagnóstico de aspergilosis invasora relacionada a COVID-19 (CAPA) se utilizaron los criterios de VerweijPE, y col. (16).

El diagnóstico de aspergilosis pulmonar crónica se realizó mediante examen micológico del material respiratorio (esputo), detección de anticuerpos para Aspergillus fumigatus, Aspergillus niger y Aspergilus flavus por la técnica de inmunodifusión en gel de agar y contrainmunoelectroforesis en agarosa. Para dichas técnicas se utilizaron antígenos comerciales (IMMY®) y/o preparados en la Unidad Micología (17). Además se realizó la valoración de las imágenes de tórax, teniendo en cuenta los criterios de Denning y col. (18).

Para el diagnóstico de candidemia se utilizaron métodos de hemocultivos automatizados (BACTEC) y hemocultivos por lisis centrifugación. Posteriormente se realizó la tipificación de las levaduras aisladas por métodos fenotípicos convencionales (17) y por espectrometría de masas (MaldiTof).

En los casos de criptococosis e histoplasmosis se emplearon las metodologías habituales de la Unidad Micología del Hospital F. J. Muñiz $(17,19,20,21)$.

\section{Análisis estadístico}

Se analizó la normalidad de las variables con el test de Shapiro-Wilks modificado, y la homogeneidad de varianzas con la Prueba F. Para objetivar la asociación entre las factores de mal pronóstico relacionados a COVID-19, las características demográficas y epidemiológicas más relevantes en los grupos de pacientes $\mathrm{VIH}+$ y $\mathrm{VIH}$ - se calculó el estadístico T de student para comparar medias y el Chi cuadrado de Pearson utilizando el programas InfoStat versión 2018 (22) y considerando un valor $p<0,05$ como estadísticamente significativo.

\section{Resultados}

Durante el período de estudio se internaron en esta institución 2837 pacientes. De ellos, 2287 tuvieron diagnóstico confirmado de COVID-19. De estos últimos,18 presentaron infecciones fúngicas pulmonares o sistémicas, ocho padecieron candidemias, cinco criptococosis meningeas, dos histoplasmosis, dos aspergilosis invasoras agudas probables (API) y una aspergilosis pulmonar crónica (APcr).

Doce eran del género masculino. La mediana para la edad fue 45 años (rango 22-70). Nueve eran pacientes $\mathrm{VIH}$ positivos, cinco hipertensos, cinco diabéticos y cuatro obesos. Catorce enfermos requirieron ingreso a unidad de cuidados intensivos durante la internación y siete fallecieron.

Las características más relevantes según el agente fúngico implicado se observan en la Tabla 1.

En relación a las candidemias, en cuatro ocasiones se aisló C. parapsilosis, en tres $C$. albicans y en una C. pelliculosa; dos enfermos fueron diagnosticados el mismo día que fallecieron, cinco fueron tratados con anidulafungina $200 \mathrm{mg}$ el primer día y luego $100 \mathrm{mg}$ /día y uno fue tratado con caspofungina $70 \mathrm{mg}$ el primer día y luego $50 \mathrm{mg} /$ día durante 14 días. Los seis evolucionaron favorablemente.

Respecto a las histoplasmosis, uno de los casos fue diagnosticado por visualización de levaduras de Histoplasma capsulatum $(\mathrm{Hc})$ en el examen directo con tinción de 


\section{Tabla 1. Características demográficas y epidemiológicas de los pacientes según la micosis} padecida

\begin{tabular}{|c|c|c|c|c|c|}
\hline & $\begin{array}{l}\mathrm{APcr}^{1} \\
\mathrm{~N}: 1\end{array}$ & $\begin{array}{l}\text { APIp }{ }^{2} \\
N: 2\end{array}$ & $\begin{array}{c}\text { Candidemia } \\
\text { N: } 8\end{array}$ & $\begin{array}{c}\text { Criptococosis } \\
\text { N: } 5\end{array}$ & $\begin{array}{c}\text { Histoplasmosis } \\
\text { N: } 2\end{array}$ \\
\hline Edad mediana & 44 & 58 & 57 & 35 & 33 \\
\hline HIV & 0 & 0 & 2 & 5 & 2 \\
\hline HTA & 0 & 1 & 4 & 0 & 0 \\
\hline DBT & 0 & 1 & 4 & 0 & 0 \\
\hline Obesidad & 0 & 1 & 3 & 0 & 0 \\
\hline LTCD4+ Mediana & $\mathrm{nr}$ & $\mathrm{nr}$ & $\mathrm{nr} \#$ & 22 & 6 \\
\hline $\begin{array}{l}\text { Neumonia asociada a } \\
\text { COVID-19 }\end{array}$ & 0 & 2 & 6 & 1 & 0 \\
\hline Fallecidos & 0 & 2 & 2 & 3 & 0 \\
\hline
\end{tabular}

${ }^{1}$ APcr: aspergilosis pulmonar crónica

${ }^{2}$ APIp: aspergilosis pulmonar invasora probable

\# se realizó LTCD4+ a dos pacientes por ser VIH positivos, tenían 13 y 350 células/ $\mu$ l

Giemsa del esputo y además presentó el antígeno urinario de galactomanano de histoplasma positivo (IMMY®) (21). El otro caso fue diagnosticado por detección de anticuerpos de Histoplasma capsulatum (contrainmunoelectroforesis) y además tuvo PCR específica positiva en sangre (PCR anidada: gen HcP 100 de Hc que codifica una proteína implicada en la infección y supervivencia de Hc) (17), también tuvo antígeno urinario positivo. El primer caso mencionado recibió anfotericina $B$ desoxicolato $0,7 \mathrm{mg} / \mathrm{kg} /$ día durante una semana y luego continuó con itraconazol $400 \mathrm{mg} /$ día (cápsulas). El segundo caso, por presentar insuficiencia renal asociada a compromiso del tubo digestivo con intolerancia digestiva, recibió anfotericina B complejo lipídico $5 \mathrm{mg} / \mathrm{kg} /$ día durante tres semanas, y luego al alta continuó con itraconazol 400mg/día (cápsulas).

Las aspergilosis invasoras probables se diagnosticaron por detección de antígeno galactomananos de Aspergillus en

\section{Figura 1. Relación entre los días transcurridos entre el diagnóstico de COVID-19 y el diagnóstico de las micosis}

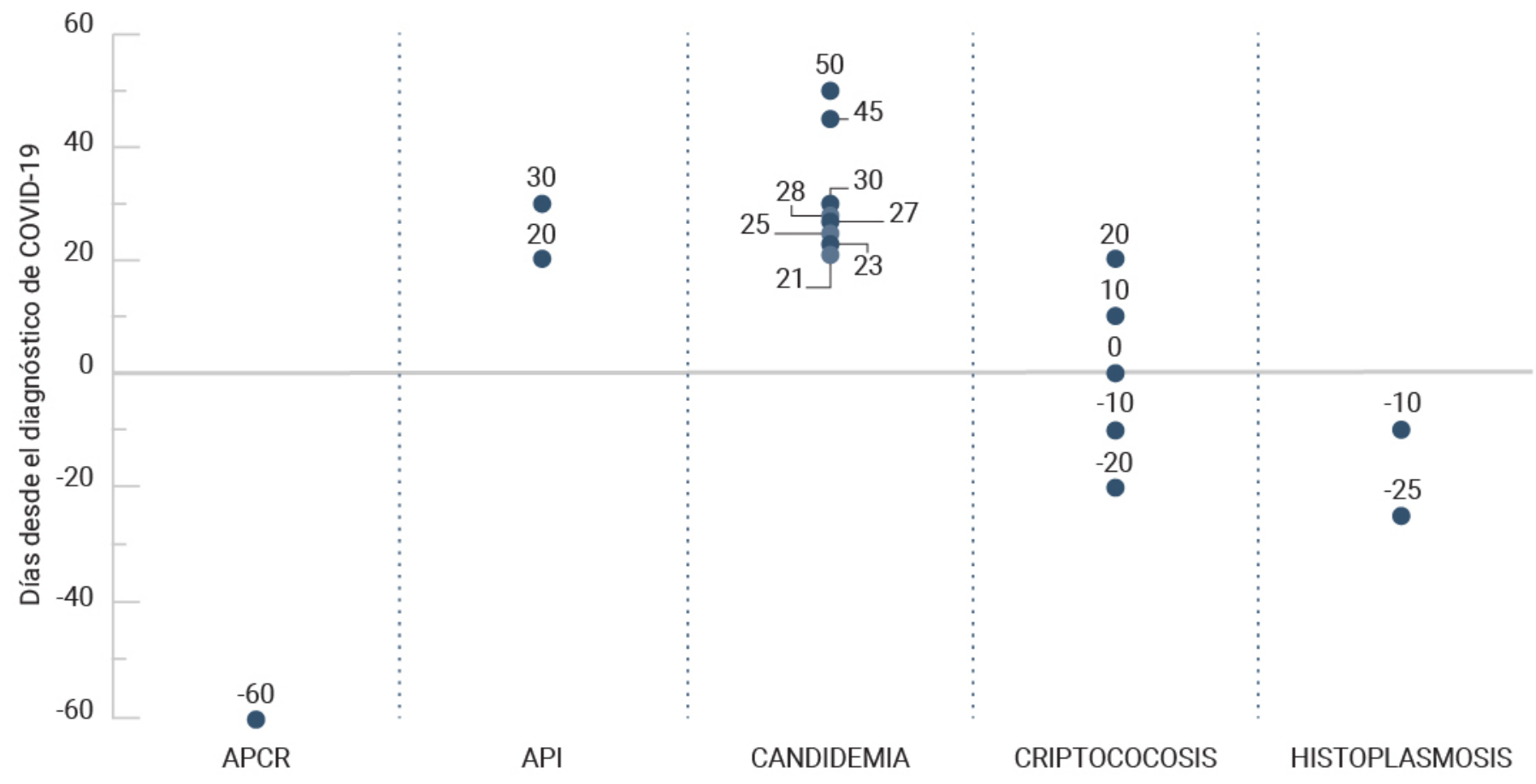


suero por la técnica de EIA (Platellia Aspergillus Bio Rad®), ambos con curva ascendente en dos determinaciones con un índice mayor a 0,5 (16); en uno de los casos el índice fue 0,15 en la primera muestra y 0,97 en la segunda, en el otro enfermo los valores fueron de 0,62 y 1,10. En ambos casos entre la primera y la segunda muestra transcurrieron $48 \mathrm{hs}$. Estos enfermos padecían neumonías bilaterales graves y se encontraban en asistencia ventilatoria mecánica (AVM). Ninguno de los dos llegó a recibir antifúngicos ya que fallecieron el día del diagnóstico. Tanto las candidemias como las aspergilosis invasoras agudas fueron diagnosticadas cursando la infección por coronavirus con estadía prolongada en terapia intensiva, en cambio la aspergilosis crónica fue diagnosticada previo a la infección viral. En las criptococosis e histoplasmosis el diagnóstico de coronavirus se realizó antes, durante o después de la infección fúngica (Figura 1).

De los nueve enfermos VIH positivos, cinco padecieron criptococosis, dos histoplasmosisy dos presentaron fungemias por Candida sp. La mediana para la edad de los pacientes VIH positivos fue de 35 años (rango 22-56), con una mediana para los linfocitos TCD4+ de 13 células/ $\mu$ l. Solo dos de estos pacientes presentaron neumonía compatible con COVID-19. Ocho enfermos ya conocían su inmunodeficiencia, pero ninguno se encontraba realizando tratamiento antirretroviral.
Los cinco enfermos con meningitis por Cryptococcus tuvieron antigenorraquias muy elevadas, evaluadas por aglutinación de partículas de látex (IMMY®), tres tuvieron un título mayor o igual a 1/10000 y dos presentaron títulos de $1 / 5000$. Tres de estos enfermos fallecieron.

En la Tabla 2 se comparan las características de los enfermos VIH positivos y negativos.

\section{Discusión}

Las características clínicas de los enfermos con COVID-19 son muy variables. Se han observado desde formas asintomáticas o leves hasta cuadros moderados o graves, requiriendo estos últimos oxigenoterapia o intubación orotraqueal con asistencia respiratoria mecánica. Los enfermos con comorbilidades como diabetes, hipertensión, obesidad y pacientes mayores de edad tienen mayor probabilidad de padecer estas formas graves $(7,8)$. Desde el inicio de la pandemia, y teniendo en cuenta todas estas características particulares, la evolución de los enfermos con VIH y COVID-19 generó un interrogante. También es desconocida su relación con las enfermedades fúngicas. La pandemia debida a gripe A (Influenza H1N1), transcurrida entre 2009 y 2010, se vinculó fuertemente a casos de aspergilosis pulmonar invasora aguda (12,

\section{Tabla 2. Características demográficas y epidemiológicas de los pacientes con COVID-19 y micosis según infección por VIH}

\begin{tabular}{|c|c|c|c|c|}
\hline & $\begin{array}{c}\text { Pacientes VIH + } \\
\text { N: } 9\end{array}$ & $\begin{array}{c}\text { Pacientes no VIH } \\
\text { N: } 9\end{array}$ & p-valor & $\begin{array}{l}\text { Estadístico T o Chi } \\
\text { cuadrado de Pearson }\end{array}$ \\
\hline Obesidad & 0 & 4 & 0,023 & 5,14 \\
\hline DBT & 0 & 5 & 0,0085 & 6,92 \\
\hline HTA & 0 & 5 & 0,0085 & 6,92 \\
\hline Neumonía asociada a COVID-19 (\#) & 2 & 7 & 0,018 & 5,56 \\
\hline $\mathrm{AVM}^{1}$ & 4 & 6 & 0,342 & 0,9 \\
\hline $\mathrm{UCl}^{2}$ & 6 & 7 & 0,598 & 0,28 \\
\hline Mortalidad & 3 & 4 & 0,628 & 0,23 \\
\hline Valor promedio de $\mathrm{VSG}^{3}(\mathrm{~mm} / \mathrm{h})$ & 59 & 75,11 & 0,283 & 1,12 \\
\hline Valor promedio de $\mathrm{PCR}^{4}$ (mg/l) & 30,8 & 178,5 & 0,1507 & 1,92 \\
\hline Valor promedio de ferritina (ng/ml) & 1022,57 & 1495 & 0,1453 & 1,54 \\
\hline Valor promedio de D-D 5 (ng/ml) & 1967,83 & 2561,4 & 0,5773 & 0,58 \\
\hline
\end{tabular}

${ }^{1}$ AVM: asistencia ventilatoria mecánica

2 UCl: unidad de cuidados intensivos

${ }^{3}$ VSG: eritosedimentación

${ }^{4}$ PCR: proteína $C$ reactiva

${ }^{5}$ D-D: dimero

\# Fueron evaluados por tomografía de tórax y/o radiografía simple de tórax 
13). Este antecedente generó preocupación en la actual pandemia por SARS-CoV-2.

Los casos de aspergilosis pulmonar invasora relacionada a COVID-19 fueron descriptos en varios países, en algunos lugares como en Italia (23) y Francia (24) con alta prevalencia y en otros como en España (25) con baja cantidad de casos.

Alrededor del 5 al 30\% de los pacientes con COVID-19 se enferman críticamente y requieren una unidad de cuidados intensivos ( $\mathrm{UCl}$ ). Como es bien sabido, los pacientes de la $\mathrm{UCl}$, especialmente los sometidos a ventilación mecánica, tienen mayor riesgo de desarrollar infecciones bacterianas o fúngicas. El COVID-19 grave está asociado con la desregulación inmune, que afecta tanto a las respuestas de células Th2 como a las de Th1, incluido el síndrome de liberación de citocinas, que contribuyen a la patología pulmonar y promueven proliferación microbiana pulmonar y una infección subsiguiente (26). Pacientes gravemente enfermos con COVID-19 tienen niveles más altos de citocinas proinflamatorias (IL-1, IL-2, IL-6, necrosis tumoral alfa) y antiinflamatorias (IL4, IL-10), menos expresión de interferón gamma CD4 y menos células CD4 y CD89 (26). Esta grave situación clínica aumenta el riesgo de infecciones fúngicas invasoras (IFI), como aspergilosis pulmonar invasora (IPA), candidiasis invasora (IC) o neumonía por Pneumocystis jirovecii (PJP) (25). En nuestra serie no tuvimos casos de PJP, probablemente debido a que el diagnóstico de esta enfermedad se realiza con muestras de lavadobroncoalveolares y dichos estudios se realizaron en bajas proporciones debido a que expone a mayor probabilidad de contagio al personal médico.

La respuesta inmune de los pacientes con COVID-19 se ha estudiado ampliamente (14). Sin embargo, la evolución y la respuesta inmune en los enfermos VIH con COVID-19 aún es desconocida y los estudios al respecto son contradictorios y han tenido limitaciones metodológicas. Por un lado, varios informes postulan que la inmunosupresión y los recuentos bajos de células CD4+ podrían actuar como un factor de protección debido a que se atenúa la posibilidad de desarrollar la tormenta de citocinas observada en pacientes con COVID-19 (28). Mientras que otros autores han demostrado que los pacientes $\mathrm{VIH}$ positivos tuvieron peor evolución que los pacientes VIH negativos (29), o no se han demostrado diferencias significativas entre ambos grupos (30). En nuestra serie pudimos observar que los factores de riesgo para COVID-19 grave, como la diabetes, la obesidad y la hipertensión, fueron estadísticamente más frecuentes en enfermos VIH negativos (Tabla 2). De la misma manera, los enfermos $\mathrm{VIH}$ negativos tuvieron mayor frecuencia de neumonías relacionadas a coronavirus. Asimismo, esto podría relacionarse con la teoría que una respuesta inmunológica exagerada parece ser perjudicial en muchos casos. Aunque para llegar a esa conclusión deben realizarse más estudios sobre este tema.

Con respecto al tratamiento específico para COVID-19, en los primeros meses de la pandemia se utilizaba lopinavirritonavir en relación al efecto in vitro demostrado en otros coronavirus $(31,32)$. Esta evidencia hacía pensar que los enfermos VIH positivos en tratamiento antirretroviral podían tener "cierta protección" contra el coronavirus, pero luego ese tratamiento fue desestimado por el ensayo SOLIDARITY (33).

Si bien la Organización Mundial de la Salud ha expresado que no existe evidencia para recomendar algún tratamiento antiviral específico, incluídos los ensayos con lopinavir/ ritonavir y darunavir/ritonavir, se ha propuesto que la combinación emtricitabina/tenofovir podría tener algún efecto inmunomodulador (34).

En este análisis observamos que todos los pacientes $\mathrm{VIH}+$ tenían una respuesta inmunológica celular deficiente debido al bajo recuento de células T CD4+. No obstante esto, solo dos enfermos presentaron una neumonía compatible con SARS-CoV-2. Esto acompaña la teoría relacionada a que una fuerte respuesta inmunológica provocaría peor evolución de la neumonía por este virus. De todas formas, al valorar los parámetros inflamatorios inespecíficos no observamos diferencias significativas en las variables de laboratorio como la proteína $\mathrm{C}$ reactiva, ferritina, dimero D o VSG. Lamentablemente no contamos en nuestro hospital con la posibilidad de cuantificar niveles de citoquinas para valorar mejor a estos dos grupos de enfermos. Por otro lado, la cantidad de pacientes evaluados no permite obtener resultados determinantes o con mayor peso estadístico, y para ello deberían realizarse estudios con una población más grande de enfermos VIH.

Existe una clara relación entre la estadía prolongada en terapia intensiva, los catéteres venosos, la diabetes y la obesidad con las fungemias por Candida $s p$. De esta manera, y por lo observado en nuestros enfermos, tenemos a la vista que en algunos casos el coronavirus ocasionó una internación prolongada y esta constituyó un factor de riesgo para desencadenar las fungemias. Otro factor de riesgo a considerar es el uso de pulsos de 
glucocorticoides. Estas drogas se utilizan en el síndrome de liberación de citocinas (SLC), una complicación grave del COVID-19, observado en el estado de hiperinflamación (35). En 2020 Lozada demostró que en el SLC, también llamado tormenta de citocinas, el aumento de los niveles de factor de necrosis tumoral a y algunas citocinas como las interleuquinas 1, 1B y 17 se asocian con edema alveolar, edema intersticial y fiebre y, a largo plazo, fibrosis pulmonar (36). Todo esto podría facilitar la progresión de la mayoría de las infecciones fúngicas debido a que muchas de estas ingresan por la vía respiratoria. Por otra parte, el desarrollo de la enfermedad aguda en COVID-19 se relaciona con la seroconversión de IgG antiviral, hecho que ocurre entre el séptimo y el décimo día de enfermedad, en el $80 \%$ de los casos (37).

Los pacientes $\mathrm{VIH}$ positivos no tenían los criterios que suelen ser de gravedad para coronavirus, pero se encontraban sin tratamiento antirretroviral con muy bajos LTCD4+, lo cual condujo a padecer las micosis oportunistas habituales en estos enfermos. Se suma a esto el retraso en la consulta debido a la pandemia. El diagnóstico de infección por coronavirus en algunos casos se observó antes, en otros después y otros en forma concomitante con el diagnóstico de las micosis (Figura 1).

A pesar de tener todos ellos una deficiente respuesta inmunológica celular, por lo que se observa en el recuento de células $\mathrm{T} \mathrm{CD4+}$, solo dos enfermos presentaron una neumonía compatible con SARS-CoV-2. Si bien no podemos aseverarlo por el número bajo de pacientes estudiados, esto acompañaría la teoría relacionada a fuerte respuesta inmunológica, peor evolución de la neumonía por este virus.

Los tres enfermos fallecidos con criptococosis meníngea tenían títulos de antigenorraquia muy elevados. En general esto se observa en pacientes que retrasan la consulta. Creemos que estos enfermos no asistieron a tiempo por temor a concurrir al hospital durante la pandemia dado el riesgo de contagio de este nuevo flagelo. Esta consulta tardía se relaciona directamente con alta carga fúngica en líquido cefalorraquídeo (Figura 2) y frecuentemente hipertensión endocraneana, lo que determina un muy mal pronóstico. Los tres enfermos fallecidos de este grupo tenían títulos superiores a 1/5000 de antigenorraquia por aglutinación de partículas de látex (IMMY®). Aunque no lo podemos aseverar, por las características descriptas, es muy probable que el deceso de estos enfermos se vincule a la criptococosis meníngea con enfermedad avanzada y no al coronavirus.
La mortalidad observada en pacientes con candidemias y COVID-19 es muy similar a la observada en candidemias no asociadas a coronavirus en Chile $(25,8 \%)$ (38) y menor a la descripta por el grupo latinoamericano de micosis invasoras (39).

Respecto a las aspergilosis, el enfermo con la forma crónica intracavitaria a pesar de padecer COVID-19 presentó una evolución favorable (Figura 3a), ni siquiera requirió oxígeno. En cambio, las formas invasoras probables (Figura $3 b$ ) tuvieron evolución fatal. De estos dos últimos casos mencionados, una paciente era obesa, hipertensa y diabética; pero el otro caso no tenía enfermedades preexistentes, solo internación prolongada en terapia intensiva.

\section{Conclusiones}

La coinfección de COVID-19 con micosis pulmonares o sistémicas fue menor al $1 \%$ en nuestra institución. A pesar de que el número de pacientes de esta serie no nos permite asegurarlo con valor estadístico, pudimos realizar las siguientes observaciones.

La estadía prolongada en terapia intensiva y los factores de riesgo asociados a esta facilitó las fungemias por Candida $s p$, que finalmente fue la micosis más frecuente.

Los casos que presentamos de histoplasmosis y criptococosis parecen relacionarse principalmente con la enfermedad avanzada por VIH y no con el COVID-19.

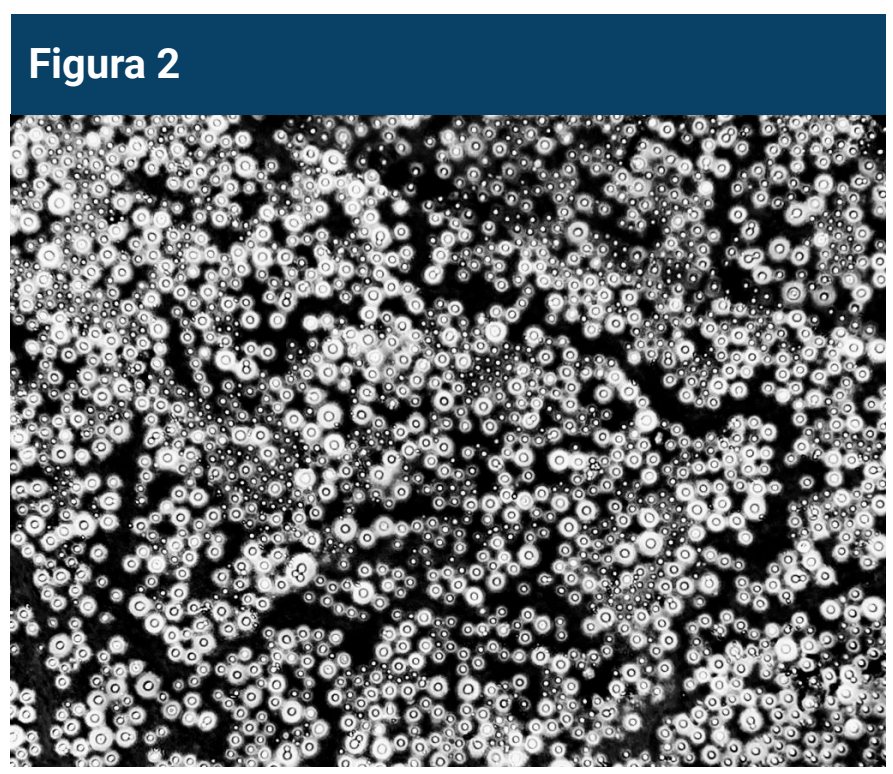

Tinta china con gran cantidad de levaduras capsuladas 


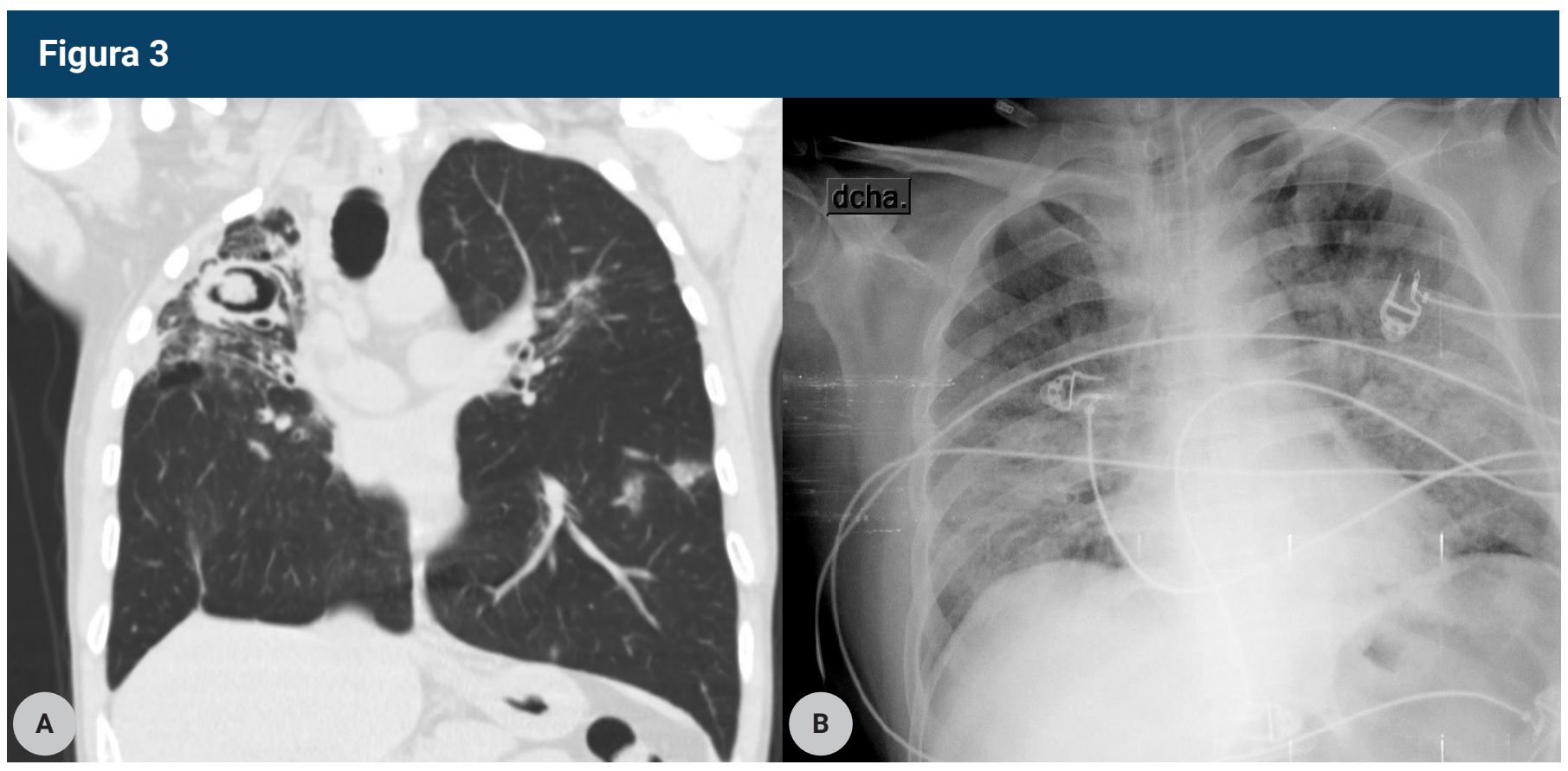

A. Tomografía de tórax, corte coronal con cavidad en lóbulo superior derecho con contenido sólido radiopaco.

B. Radiografía de tórax frente con opacidades alveolares multifocales bilaterale.

El componente inflamatorio y la respuesta inmunológica de los pacientes con aspergilosis pulmonar invasora probablemente tengan relación con la neumonía por coronavirus.

Los enfermos con factores de riesgo como diabetes, obesidad e hipertensión arterial con neumonía grave por coronavirus se relacionan más con micosis invasoras como aspergilosis o candidiasis que los enfermos $\mathrm{VIH}$ positivos con niveles bajos de LTCD4+. 


\section{Bibliografía}

1. Informe SARSCOV2, Marzo 2020. Sociedad Argentina de virología - AAM. Available from: https://aam.org. ar/src/img_up/22032020.0.pdf

2. Guan Y., Zheng B.J., He Y.Q., Liu X.L., Zhuang Z.X., Cheung C.L., et al. Isolation and characterization of viruses related to the SARS coronavirus from animals in southern China. Science. 2003; 302:276-278.

3. World Health Organization. Middle East respiratory syndrome coronavirus (MERS-CoV) (http://www.who. int/emergencies/mers-cov/en/. opens in new tab).

4. World Health Organization. Coronavirus Disease (COVID-19) pandemic Available from: https:// www.who.int/emergencies/diseases/novelcoronavirus-2019. Accessed 13 May 2020

5. Weekly epidemiological update - 28 September 2020 Data as received by WHO from national authorities, as of 27 September 2020. https://www.who.int/ publications/m/item/weekly-epidemiologicalupdate---28-september-2020

6. Ministerio de salud de la nación. Informe del 30-09-20-reporte-vespertino-covid-19. https://www. argentina.gob.ar/coronavirus/informes-diarios/ reportes/septiembre2020

7. Guan WJ, Ni ZY, Hu Y, Liang WH, Ou CQ, He JX, et al. Clinical Characteristics of Coronavirus Disease 2019 in China. N Engl J Med 2020; 382 (18):1708-20. doi: 10. 1056/NEJMoa2002032.

8. The epidemiological characteristics of an outbreak of 2019 novel coronavirus diseases (COVID-19) China, 2020. China CDC Weekly. 2020; 2:10.

9. Gangneux JP, Bougnoux ME, Dannaoui E, Cornet $M$, Zahar JR. Invasive fungal diseases during COVID-19: We should be prepared. J Mycol Med. 2020 Jun;30(2):100971. doi: 10.1016/j. mycmed.2020.100971. Epub 2020 Apr 6. PMID: 32307254; PMCID: PMC7136887.

10. Song, G., Liang, G. \& Liu, W. Fungal Co-infections Associated with Global COVID-19 Pandemic: A Clinical and Diagnostic Perspective from China. Mycopathologia 185, 599-606 (2020). https://doi. org/10.1007/s11046-020-00462-9

11. Menon AA, Berg DD, Brea EJ, Deutsch AJ, Kidia KK, Thurber EG, et al. A Case of COVID-19 and Pneumocystis jirovecii Coinfection. Am J Respir Crit Care Med. 2020;202(1):136-8.

12. Vanderbeke L, Spriet I, Breynaert C, Rijnders BJA, Verweij PE, Wauters J. Invasive pulmonary aspergillosis complicating severe influenza: epidemiology, diagnosis and treatment. Curr Opin Infect Dis. 2018;31(6):471-480. doi:10.1097/ QC0.0000000000000504

13. Schauwvlieghe AFAD, Rijnders BJA, Philips N, Verwijs R, Vanderbeke L, Van Tienen C, et al. DutchBelgian Mycosis study group. Invasive aspergillosis in patients admitted to the intensive care unit with severe influenza: a retrospective cohort study. Lancet Respir Med. 2018 Oct;6(10):782-792. doi: 10.1016/ S2213-2600(18)30274-1

14. García LF. Immune Response, Inflammation, and the Clinical Spectrum of COVID-19. Front Immunol. 2020 Jun 16;11:1441. doi: 10.3389/fimmu.2020.01441. PMID: 32612615; PMCID: PMC7308593.

15. Del Amo J, Polo R, Moreno S, Díaz A, Martínez E, Arribas $\mathrm{J}$, et al. Incidencia y gravedad de COVID19 en personas $\mathrm{VIH}$ positivas con terapia antirretroviral. Ann Intern Med 2020. doi: 10.7326/M20-3689. PMID: 32589451

16. Verweij, P.E., Rijnders, B.J.A., Brüggemann, R.J.M. et al. Review of influenza-associated pulmonary aspergillosis in ICU patients and proposal for a case definition: an expert opinion. Intensive Care Med 46, 1524-1535 (2020). https://doi.org/10.1007/s00134020-06091-6

17. Manual de procedimientos Unidad Micología Hospital de Infecciosas Francisco J. Muñiz, centro de referencia de la Ciudad Autónoma de Buenos Aires, versión 2020.

18. Denning DW, Cadranel J, Beigelman-Aubry C, Ader F, Chakrabarti A, Blot S, Ullmann AJ, Dimopoulos G, Lange C; European Society for Clinical Microbiology and Infectious Diseases and European Respiratory Society. Chronic pulmonary aspergillosis: rationale and clinical guidelines for diagnosis and management. Eur Respir J. 2016 Jan;47(1):45-68. doi: 10.1183/13993003.00583-2015. PMID: 26699723.

19. Arechavala A, Negroni R, Messina F, Romero M, Marín E, Depardo R, Walker L, Santiso G. Cryptococcosis in an Infectious Diseases Hospital of Buenos Aires, Argentina. Revision of 2041 cases: Diagnosis, clinical features and therapeutics. Rev Iberoam Micol. 2018 Jan-Mar;35(1):1-10. doi: 10.1016/j.riam.2017.04.003. Epub 2017 Nov 10. PMID: 29129578.

20. Messina FA, Corti M, Negroni R, Arechavala A, Bianchi M, Santiso G. Histoplasmosis en pacientes con SIDA sin manifestaciones cutáneomucosas [Histoplasmosis in AIDS patients without tegumentary manifestations]. Rev Chilena Infectol. 2018;35(5):560-565. Spanish. doi: 10.4067/s0716- 
10182018000500560 . PMID: 30725004.

21. Messina FA, Marin E, Caceres DH, Romero M, Depardo R, Priarone MM, Rey L, Vázquez M, Verweij PE, Chiller TM, Santiso G. Coronavirus Disease 2019 (COVID-19) in a Patient with Disseminated Histoplasmosis and HIV-A Case Report from Argentina and Literature Review. J Fungi (Basel). 2020 Nov 10;6(4):E275. doi: 10.3390/jof6040275. PMID: 33182836.

22. Di Rienzo J.A., Casanoves F., Balzarini M.G., Gonzalez L., Tablada M., Robledo C.W. InfoStat versión 2020. Centro de Transferencia InfoStat, FCA, Universidad Nacional de Córdoba, Argentina. URL http://www. infostat.com.ar

23. Bartoletti M, Pascale R, Cricca M, Rinaldi M, Maccaro A, Bussini L, et al. Epidemiology of invasive pulmonary aspergillosis among COVID-19 intubated patients: a prospective study. Clin Infect Dis. 2020.

24. Gangneux JP, Reizine $F$, Guegan $H$, Pinceaux $K$, Le Balch P, Prat E, et al. Is the COVID-19 Pandemic a Good Time to Include Aspergillus Molecular Detection to Categorize Aspergillosis in ICU Patients? A Monocentric Experience. J Fungi (Basel). 2020;6(3).

25. Pemán J, Ruiz-Gaitán A, García-Vidal C, Salavert M, Ramírez $\mathrm{P}$, Puchades $\mathrm{F}$, et al. Fungal co-infection in COVID-19 patients: Should we be concerned? Rev Iberoam Micol. 2020 Sep 14:S1130-1406(20)300310. doi: 10.1016/j.riam.2020.07.001.

26. Vizcarra P, Pérez-Elías MJ, Quereda C, Moreno A, Vivancos MJ, Dronda F, et al. Description of COVID-19 in HIV-infected individuals: a single-centre, prospective cohort. Lancet HIV. agosto de 2020;7(8):e554-64.

27. Aydin OA, Karaosmanoglu HK, Yasar KK. HIV/SARSCoV-2 coinfected patients in Istanbul, Turkey. J Med Virol. 2020;92(11):2288-90.

28. de la Rica R, Borges M, Gonzalez-Freire M. COVID-19: In the Eye of the Cytokine Storm. Front Immunol. 2020 Sep 24;11:558898. doi: 10.3389/fimmu.2020.558898. PMID: 33072097; PMCID: PMC7541915.

29. Childs K, Post FA, Norcross C, Ottaway Z, Hamlyn E, Quinn K, et al. Hospitalized Patients With COVID-19 and Human Immunodeficiency Virus: A Case Series. Clin Infect Dis. 27 de mayo de 2020;ciaa657.

30. Gudipati S, Brar I, Murray S, McKinnon JE, Yared N, Markowitz N. Descriptive Analysis of Patients Living with HIV Affected By COVID-19. JAIDS J Acquir Immune Defic Syndr [Internet]. 13 de julio de 2020 [citado 4 de octubre de 2020];Publish Ahead of Print. Disponible en: https://journals.Iww.com/10.1097/ QAI.0000000000002450

31. Chu CM, Cheng VC, Hung IF, et al. Role of lopinavir/ ritonavir in the treatment of SARS: initial virological and clinical findings. Thorax 2004; 59:252-256.

32. Chen F, Chan KH, Jiang Y, Kao RY, Lu HT, Fan KW, et al. In vitro susceptibility of 10 clinical isolates of SARS coronavirus to selected antiviral compounds. J Clin Virol. 2004 Sep;31(1):69-75. doi: 10.1016/j. jcv.2004.03.003. PMID: 15288617; PMCID: PMC7128415.

33. Repurposed antiviral drugs for COVID-19 -interim WHO SOLIDARITY trial results WHO Solidarity trial consortium, Hongchao Pan, Richard Peto, Quarraisha Abdool Karim, Marissa Alejandria, Ana Maria Henao-Restrepo, César Hernández García et al. medRxiv 2020.10.15.20209817; doi: https://doi. org/10.1101/2020.10.15.20209817

34. Randomized Clinical Trial for the Prevention of SARSCoV-2 Infection (COVID-19) in Healthcare Personnel (EPICOS) Consultado en https://clinicaltrials.gov/ct2/ show/NCT04334928? cond= Randomized+clinical +trial+for+the tprevention+of+SARS-CoV2+infection+\%28COVID

35. Wu C, Chen X, Cai Y, Xia J, Zhou X, Xu S, et al. Risk factors associated with acute respiratory distress síndrome and death in patientes with coronavirus disease. JAMA Intern Med 2020; 180 (7): 934-943

36. Lozada I, Nuñez C. COVID 19: respuesta inmune y perspectivas terapéuticas. Rev perú Med Exp Salud Pública. 2020; 27(4):312-9

37. Fu $\mathrm{Y}$, Cheng $\mathrm{Y}$, Wu $\mathrm{Y}$. Understanding SARS CoV2 mediated inflammatory responses: From mechanisms to potential therapeutic tools. Virol Sin 2020; doi 10.1007/5 12250 - $020-00207-4$

38. Santolaya ME, Thompson L, Benadof D, Tapia C, Legarraga P, Cortés C, et al. (2019) A prospective, multi-center study of Candida bloodstream infections in Chile. PLoS ONE 14(3): e0212924. https://doi. org/10.1371/journal.pone.0212924

39. Nucci $M$, Queiroz-Telles $F$, Alvarado-Matute $T$, Tiraboschi IN, Cortes J, Zurita J et al. Latin American Invasive Mycosis Network. Epidemiology of candidemia in Latin America: a laboratory-based survey. PLoS One. 2013;8(3):e59373. doi: 10.1371/ journal.pone.0059373. Epub 2013 Mar 19. PMID: 23527176; PMCID: PMC3601956. 


\section{Fungal infections in patients with COVID-19}

On December 2019 a new coronavirus (SARS-CoV2) result in atypical pneumonía epidemic, it was identified in Wuhan China and it was called COVID-19. Then on March 11 was declared pandemic by the WHO.

Until September 30, 2020 in Argentina 751,001 cases and more than 16,937 deaths have been confirmed. The frequency and impact of co-infections affecting SARSCov2 infected patients has been studied with the advance of the pandemic. Among those due to fungi are Candida sp fungemias, invasive aspergillosis, endemic systemic mycoses, and pneumocystosis.

We present the different mycosis-COVID-19 co-infections that were assisted in F. J. Muñiz Hospital between April and September of this year and review the characteristics of these infections in patients with and without AIDS is carried out.

In this period, 2,837 patients were admitted in the Muñiz hospital, 2,287 had a confirmed diagnosis of COVID-19.

Co-infection of COVID-19 with pulmonary or systemic mycoses was less than $1 \%$.

Eighteen patients had pulmonary or systemic fungal infections. Eight suffered from candidemia, five meningeal cryptococcosis, two histoplasmosis, two probable acute invasive aspergillosis, and one chronic pulmonary aspergillosis.

Prolonged stay in intensive care facilitated fungemia due to Candida sp. Histoplasmosis and cryptococcosis cases seem to be related to advanced HIV disease and not to COVID-19.

Patients with a high baseline inflammatory component with severe coronavirus pneumonia are more associated with invasive mycoses than HIV-positive patients with low levels of LTCD4 +.

Keywords: Mycosis and COVID-19, Candidemia and COVID-19, SARS-CoV-2 and mycosis. Invasive aspergillosis. 